\title{
PIC and Sensors Based Automatic Car Washing System
}

\author{
Ei Ei Aung \\ Department of Electronic \\ Engineering \\ Technological University (Lashio), \\ Myanmar
}

\begin{abstract}
This paper describes the design and implementation of automatic car washing system for the exterior of the vehicles using PIC16F877A microcontroller. There are five main parts to implement the system; moving, washing, foaming, brushing and cleaning. To accomplish the system, conveyor, LDR sensor circuits, motor driver circuits, pumps and motors are comprised. The conveyor is used to carry the vehicle and moved from step to step. The sensor circuit sensed and input data to the PIC. The outputs of PIC are commands to drive motors and pumps.

Keywords: automatic; car washing; PIC; conveyor; LDR; motors; pumps
\end{abstract}

\section{INTRODUCTION}

Car washing system is a facility used to clean the exterior and, in some cases, the interior of motor vehicles. As the transportation section is developed, the most common problem often encountered while cleaning these vehicle is time consumption. Then automatic car washing system is an attractive solution to the problem.

Automatic car washing machine consists of tunnel- like building. In this system, a car enters a washing station and automatically gets cleaned up. This system design can provide reliability, safety and reducing time consumption. This paper is mentioned on the basic research of developing on car washing and can be realized the operation of the Automatic Car Washing System. This system is controlled by using programmable interface controller (PIC). There are five steps involved in car washing system namely moving, washing, foaming, brushing and cleaning. The various components used in this project are the conveyor belt, sensors, buzzer, dc motors, pumps and brushes.

Using this automatic car washing system, many cars can be washed and it will save time, energy and manpower. Automation of the process is having its own significance with the advancement of science and technology. As the technology is upgrading day by day and everyday it creating life easier, so take advantage of these advancements. Such systems can be installed anywhere such as malls, airports, railway stations etc. This process is based on cyclic process which provides more than $95 \%$ cleaning.

In this paper, there are mainly five sections to implement this control system. Firstly the system is introduced. The second section describes the system description of overall control system, and some detailed features of the Automatic Car Washing System. The third and fourth sections present the software and hardware implementation, test and result of the overall system. Conclusion is mentioned in the fifth section.

\section{SYSTEM DESCRIPTION}

The technical characteristic of an Automatic Car Washing System are designed as follows. The five processes are moving, washing, foaming, brushing and cleaning. Firstly the conveyor is on and the car is entered to the station. When the car reaches on LDR sensor1, sensor senses and the pump will spray the car with water. When the car reaches on LDR sensor2, sensor senses and the pump will spray the car with detergent water. When the car reaches on LDR sensor3, sensor senses and the motor will brush the car with brusher. Finally, when the car reaches on LDR sensor4, the pump will clean the car for 10 seconds. After finishing all processes, the buzzer will sound for 2 seconds and then the conveyor belt will stop.

The block diagram of Microcontroller based Automatic Car Washing System is shown in Figure 1.

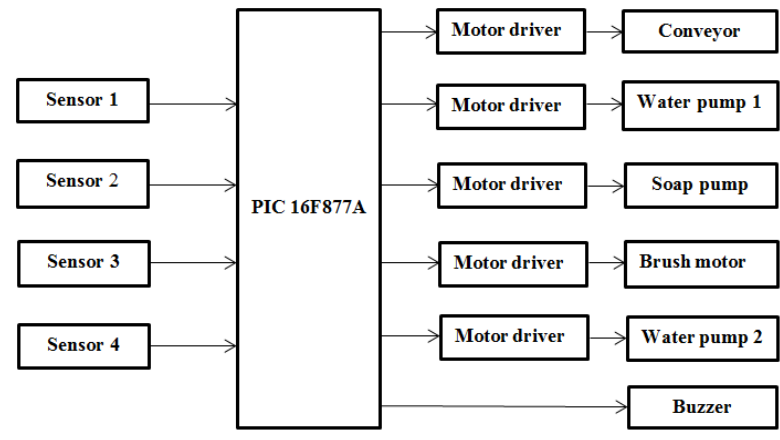

Figure 1. Block diagram of Automatic Car Washing System

\subsection{Microcontroller}

The major difference of a microcontroller compared to a microprocessor and microcomputer is that microcontroller consists of central processing unit (CPU), memory devices (ROM and RAM), input and output ports and timer embedded into a single ship. PIC 16F877A is mainly used to implement the automatic car washing design. It has many I/O ports such as PORTA, PORTB, PORTC, PORTD and PORTE. Speed of $4 \mathrm{MHz}$ crystal is used to carry out the algorithm. Six pins of PORTB is used as output pins to the motors and pumps, and other pins of PORTD are used as input pins to sense the car and input data to the PIC.

\subsection{Sensor and Relay Driver Circuits}

The $12 \mathrm{~V}$ supply voltage supplies to all sensor circuits, motors, pumps and conveyor belt and then 5V supplies the PIC. When the car reaches on LDR sensor1, sensor senses and the incoming signal reaches to the base of transistor Q1 which collector is connected to the relay. The common point of relay is connected to the input pin of the PIC RDO. At the time the signal reaches the input pin RD0, the program runs. The signal from the output pin RB0 and RB1 enter through the base of transistor Q5 and Q6 which collector are connected to 
the relay. The $12 \mathrm{~V}$ supply voltage supplies to the common point of relay at the output. When the relay on, the conveyor belt and pump1 starts working. There are four sensor circuits with four motors or pumps respectively.

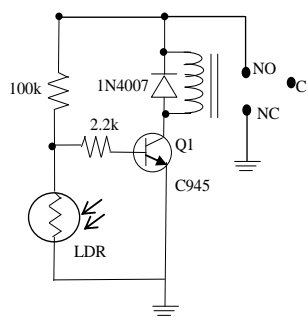

Figure 2. LDR sensor circuit

Design for one sensor circuit,

To saturate the transistor, $\mathrm{V}_{\mathrm{B}}$ must greater than $\mathrm{V}_{\mathrm{BE} \text { (sat) }}$. Using voltage divider formula, $\mathrm{V}_{\mathrm{B}}=\left(\mathrm{R}_{\mathrm{LDR}} /\left(\mathrm{R}_{1}+\mathrm{R}_{\mathrm{LDR}}\right)\right) \times \mathrm{V}_{\mathrm{CC}}$, let $\mathrm{R} 1=100 \mathrm{k} \Omega$. When LDR resistance is reached $10.4 \mathrm{k} \Omega, \mathrm{V}_{\mathrm{B}}$ is $1.13 \mathrm{~V}$ and $\mathrm{I}_{\mathrm{B}}$ can calculate from $\mathrm{I}_{\mathrm{C}}\left(\mathrm{I}_{\text {relay }}\right) / \mathrm{h}_{\mathrm{FE}}$ and applying Kirchhoff's voltage law, $R_{B}=\left(V_{B}-V_{B E(s a t)}\right) / I_{B}$, the base resistor is $2.2 \mathrm{k} \Omega$.

Design for relay driver circuit,

When PIC output is $5 \mathrm{~V}, \mathrm{~V}_{\mathrm{BE}(\text { sat })}$ is $0.8 \mathrm{~V}$ and $\mathrm{I}_{\mathrm{B}}$ can calculate from $\mathrm{I}_{\mathrm{C}}\left(\mathrm{I}_{\text {relay }}\right) / \mathrm{h}_{\mathrm{FE}}$ and applying Kirchhoff's voltage law, $R_{B}=\left(V_{B}-V_{B E(\text { sat })} / I_{B}\right.$, the base resistor is $28 \mathrm{k} \Omega$ and choose 27 $\mathrm{k} \Omega$ as a standard value.

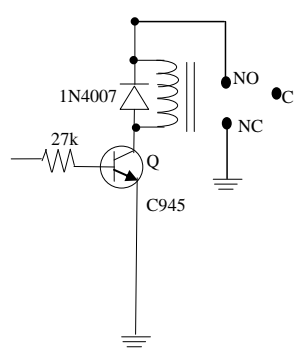

Figure 3. Relay driver circuit

\subsection{Overall Circuit Diagram}

The overall circuit diagram is illustrated in Figure 4.

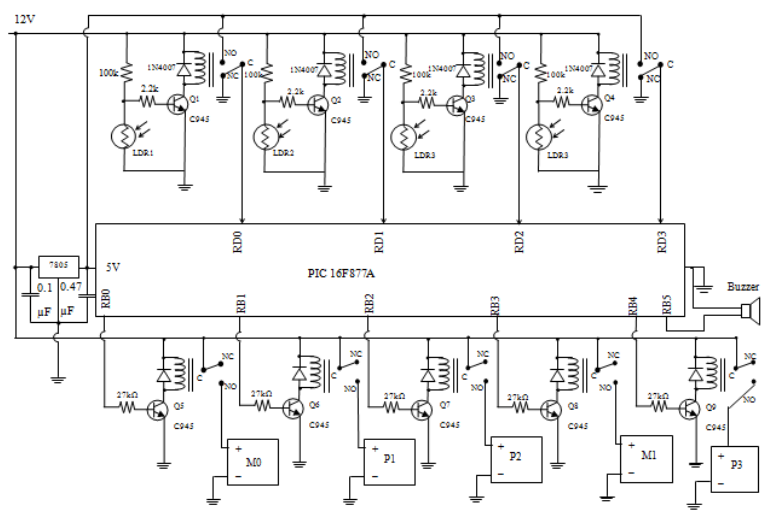

Figure 4. Overall Circuit of Automatic Car Washing System

In this circuit design, there are five main portions to implement the system. They are moving washing, foaming, brushing and cleaning. Then buzzer is alarm to know the process is complete. Based on the overall system design, the simulation result is implemented by using mikroC Pro for PIC and Proteus software.

\section{SOFTWARE IMPLEMENTATION}

\subsection{Flowchart of the System}

When the car reaches on LDR sensor1, the conveyor is on and the water pump1 is on .When LDR sensor2 is active, the soap pump is on. When LDR sensor3 is detected the car, the brush motor is active to wash the car with brushes. Finally, when the car reaches on LDR sensor4, the water pump2 is on to clean the car for 10 seconds. After finishing all processes, the buzzer will sound for 2 seconds and then the conveyor belt will stop.

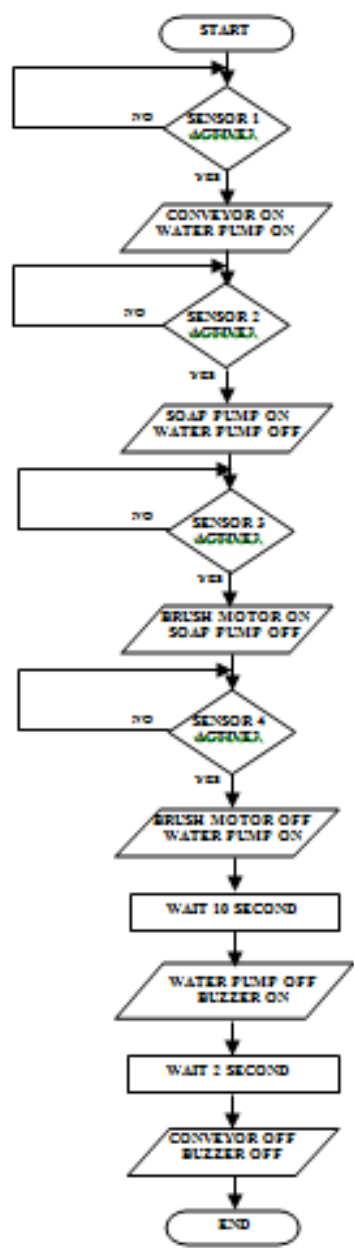

Figure 5. Flowchart of the system

\subsection{Simulation and Compiling}

Firstly, the $\mathrm{C}$ program to test the simulation must be written in the Notepad as C file. And then using mikroC Pro for PIC complier software, program must be compiled. When the result is succeed, there are many files that created by the software. Among them, the HEX file is needed to link with the PIC. The circuit diagram with the PIC microcontroller is drawn and compiled with the HEX file. Finally simulation test is implemented with the Proteus software. If the result is satisfied, it is downloaded to PIC using Easy PIC kit. Figure 6 show the test result of compiled code is succeeded. 


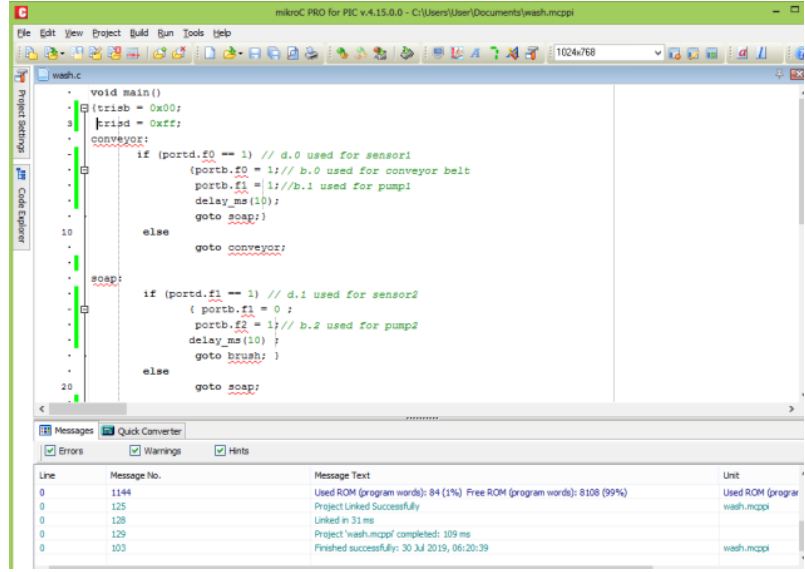

Figure 6. Testing software implementation

\section{TEST AND RESULT}

When the car reaches on LDR sensor1, the conveyor is on and the water pump1 also starts for washing as shown in Figure 7.

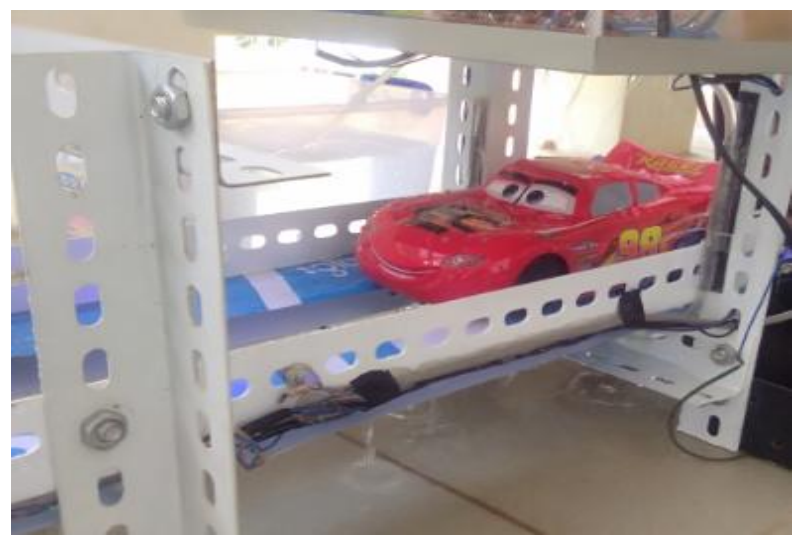

Figure 7. Washing the car

When the car reaches on LDR sensor2, the soap pump is on for foaming as shown in Figure 8.

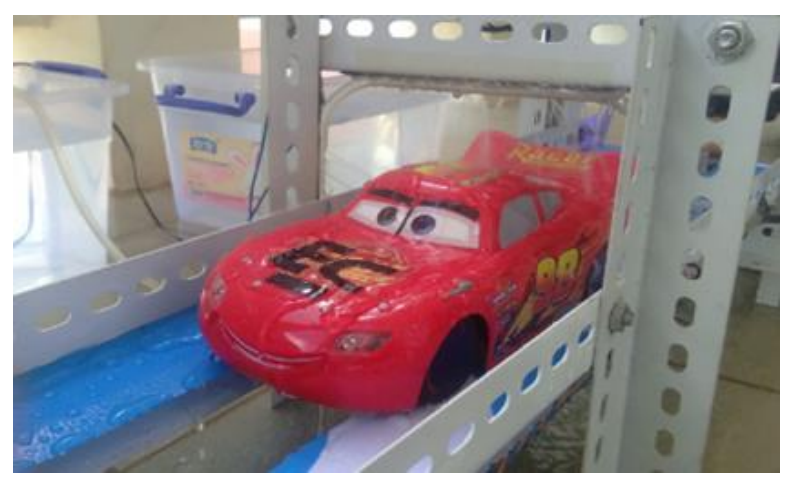

Figure 8. Foaming the car with detergent liquid

When the car reaches on LDR sensor3, the brush motor is on for brushing as shown in Figure 9.

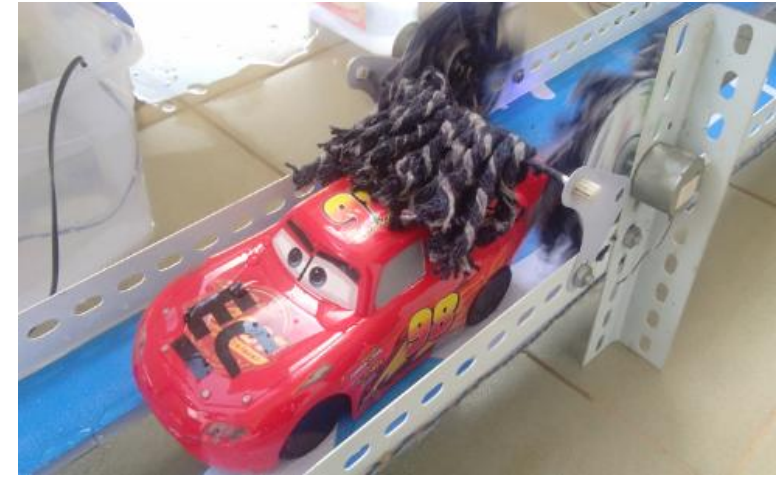

Figure 9. Brushing the car

When the car reaches on LDR sensor4, the water pump 2 is active for cleaning as shown in Figure 10.

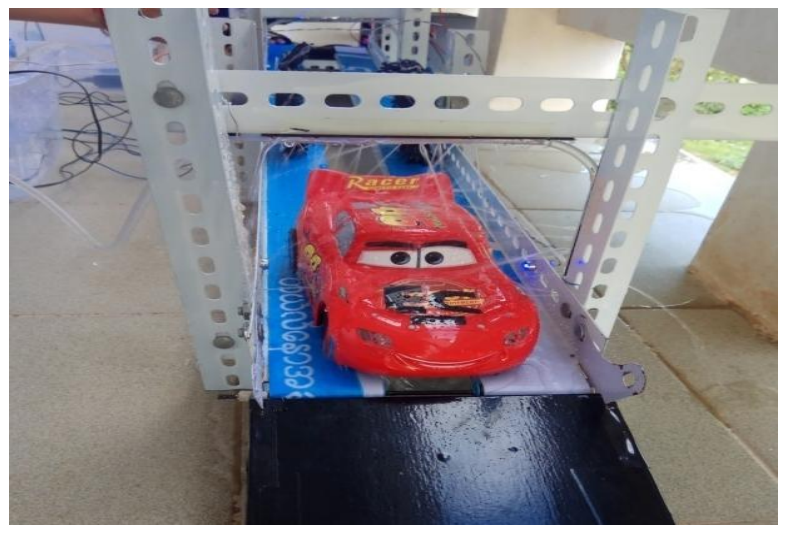

Figure 10. Final cleaning the car

Complete system design is accomplished as shown in Figure 11. There are four sections except conveyor and their respective tanks; two water tanks and one soap tank.

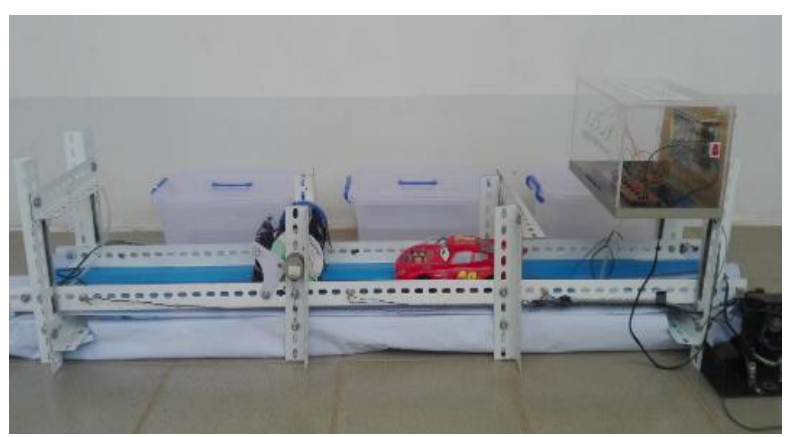

Figure 11. Complete system of Automatic Car Washing System

\section{CONCLUSION AND DISCUSSION}

Car washing is a service that becomes essential role in most of cities currently in developing country. Automation was created to reduce manpower, to make production process easier and increase its quantities several times. The system aim to develop the required demand on reduces time consumption, less cost and reduces human errors. C language is used because of the short learning curve that most students have the basic of the $\mathrm{C}$ language, and now its wide distribution and its general- purpose nature have to be studied and linked with the PIC microcontroller technology. In addition drying and polishing can be extended. And cleaning of the interior in the vehicles is also expanded. 


\section{ACKNOWLEDGMENTS}

The author would like to thank to her family and partners who have contributed towards development of this work.

\section{REFERENCES}

[1] D.W Smith. 2002. "PIC in practice", Gutenberg Press Ltd.

[2] Anonymous, 2003, "PIC16F87X Datasheet", Microchip Technology Inc.

[3] Lucio Di Jasio,Tim Wilmshurst, Dogan Ibrahim, John Morton, Martin P. Bates, Jack Smith, D. W. Smith, Chuck Hellebuyck. 2008. " PIC Microcontrollers: know it all", Elsevier Inc.
[4] Thomas L. Floyd.2007. "Electronic Fundamentals: Circuit, Devices and Application".

[5] Janik, A. Kupiec. 2007. "Trends in Modern Car Washing” Polish J. of Environ Stud.Vol.16, No.6.

[6] M., Amir Hossein Daei Sorkhabi, "Manufacturing of Full Automatic Carwash Using With Intelligent Control Algorithms", IEEE Vol: 7, No: 3, 2013.

[7] Muhammadali V.V. 2010. Programmable Controller, Principles and Applications, "Seminar on Automatic Car Washing System".

[8] Irfan Anjum, Tarif Hussain, M. Fasial Mafeed. 2018. " PLC Based Automatic Car Wash System". 